\title{
Changes in CMDP and DPOAE during acute increased inner ear pressure in the guinea pig
}

\author{
W. L. Valk $\cdot$ H. P. Wit $\cdot$ F. W. J. Albers
}

Received: 29 June 2006/ Accepted: 4 September 2007/Published online: 28 September 2007

(C) Springer-Verlag 2007

\begin{abstract}
During and after an increase of inner ear pressure, induced by injection of artificial perilymph, the $2 f_{1}-f_{2}$ and $f_{2}-f_{1}$ distortion products (DPs) in cochlear microphonics (CM) and otoacoustic emissions (OAE) were recorded in the guinea pig. An inner pressure increase of $\sim 600$ Pa gave only small changes in CMDP and DPOAE. Along with a decrease in $f_{1}$ amplitude, a small decrease in amplitude of the $2 f_{1}-f_{2}$ and a small increase in the $f_{2}-f_{1}$ were measured in CM. This matches a shift from a symmetrical position of the operating point for hair cell transduction, leading to an increase in even-order distortion and a decrease in odd-order distortion. Similar, a decrease in $2 f_{1}-f_{2}$ DPOAE was expected. This might be the case at the generation sites but this effect was then more than compensated for by a better middle ear transfer, accounting for the increase of $0.4 \mathrm{~dB}$ of the $2 f_{1}-f_{2}$ DPOAE amplitude. In conclusion, changes of overall inner ear fluid pressure have minor effects on cochlear function. This is a relevant finding for further understanding of diseases with changed inner ear fluid volumes, as Ménière's.
\end{abstract}

Keywords Perilymph - Injection - Inner ear pressure . $2 f_{1}-f_{2} \cdot f_{2}-f_{1} \cdot$ CMDP $\cdot$ DPOAE $\cdot$ Guinea pig

\section{Introduction}

The cochlea is a non-linear mechano-electrical transducer that transforms sound pressure waves into electrical

W. L. Valk $(\bowtie) \cdot$ H. P. Wit · F. W. J. Albers

Department of Otorhinolaryngology,

University Hospital Groningen, PO Box 30.001,

9700 RB Groningen, The Netherlands

e-mail: w.l.valk@kno.umcg.nl information, the so-called mechanoelectric transduction (MET). The reverse electromechanical transduction is performed by the outer hair cell $(\mathrm{OHC})$ which mechanically influences the vibration of the cochlear partition [7]. When the cochlea is stimulated by two tones, distortion products (DPs) can be measured in cochlear microphonic potentials (CMDP) and in the external auditory meatus as otoacoustic emissions (DPOAE). Both microphonic and acoustic DPs are considered to be reflections of OHC electromotility in the cochlea. Obviously, it is of interest to find a relation between these two functional modalities of the cochlea and the OHC in particular.

In this study the most prominent DPs, the $2 f_{1}-f_{2}$ and $f_{2}-f_{1}$, have been observed while increasing inner ear pressure. This was done by rapid injection of artificial perilymph into the scala tympani of the guinea pig's cochlea. Measurement of specific DPs may describe changes in gain and operating point of the non-linear cochlear amplifier. Furthermore, transfer characteristics of forward and backward transmission may be clarified by using CMDP and DPOAE [17]. These data could help gaining insight into pathophysiological mechanisms involved in pathologically increased inner ear pressures.

When pressure variation is in the normal physiological range, this does not influence cochlear function as measured by compound action potentials in the guinea pig [2]. In humans, during activities like coughing or sneezing, perilymphatic pressure may undergo substantial fluctuations up to $10 \mathrm{mmHg}(1.4 \mathrm{kPa})$.

An increased pathologic inner ear pressure may be the result of an increased intracranial pressure since the perilymphatic space is linked to the subarachnoid space via the cochlear aqueduct. Human OAE have been proven to be very sensitive to modifications of intracranial pressure [10]. Another clinical example of increased inner ear pressure or 
volume with an accompanying change of cochlear function is the endolymphatic hydrops in patients with Ménière's disease.

In a previous study [11], we measured solely the $2 f_{1}-f_{2}$ DPOAE's in response to microinjection of artificial perilymph into the cochlea. Large changes of $\sim 500 \mathrm{~Pa}$ in overall inner ear pressure gave only a small mean change of $0.7 \mathrm{~dB}$ in $2 f_{1}-f_{2}$ DPOAE amplitude. The magnitude of change could (partly) be explained by a change in oval window stiffness. In this study, the same experimental setup was used, with the addition of the simultaneous recording of the $2 f_{1}-f_{2}$ and $f_{2}-f_{1}$ CMDP. We attempt to establish a better understanding of increased inner ear pressure effects on DPs in both CM potentials and OAE's.

\section{Materials and methods}

Experiments were performed in eight guinea pigs (Harlan Laboratories, The Netherlands; body weight 350-450 g) with a positive Preyer reflex. Animal care and use were in accordance with the principles of the declaration of Helsinki and approved by the animal experiment committee (protocol number 3047/3103).

General anesthesia was induced by intramuscular administration of ketamine/xylazine (60/3.5 mg/kg). Maintenance doses of the anesthetic were administered every hour. Muscle relaxation was obtained with succinylcholine $(2.5 \mathrm{mg} / \mathrm{kg})$. The animals were artificially ventilated through a tracheostoma (Columbus Instruments, model 7950). Body temperature was maintained at $38^{\circ} \mathrm{C}$ with a heating blanket. Heart rate was monitored by skin electrodes placed on both sides of the thorax. The animal's head was kept in a stationary position by means of a steel bolt fixed to the skull with dental cement. Following a retroauricular incision, the bulla and external auditory canal were exposed. Subsequently, the bulla was opened equalizing middle ear pressure to normal air pressure [17]. By opening of the bulla, the round window was exposed. Through the round window membrane, the tip of a double-barreled micropipette was inserted into scala tympani. The double-barreled micropipettes were drawn from borosilicate glass (1.5/ $0.84 \mathrm{~mm}$ diameter per barrel) and the tips were beveled (Narishige EG-40). The total tip diameter was around $60 \mu \mathrm{m}$, which is a compromise between a low enough flow resistance for fluid injection and tip smallness. One barrel of the pipette was used to measure inner ear pressure (WPI 900A micropressure system). Through the other barrel, artificial perilymph [9] was injected with a constant flow rate by applying a controllable pneumatic pressure to the barrel end. The injected volume was measured as the displacement of the fluid meniscus in the pipette, for which the inner diameter is precisely known $(0.84 \mathrm{~mm})$. The fluid injection rate was calculated as the total injected volume divided by the total injection time.

During the experiment the DPOAE's were continuously measured using an Etymotic ER-10C DPOAE probe system. The two primary frequencies were set at $6 \mathrm{kHz}\left(f_{1}\right)$ and $7.4 \mathrm{kHz}\left(f_{2}\right)$, meaning a $f_{2} / f_{1}$-ratio of 1.23 . The lowlevel intensities were set at, respectively, $65 \mathrm{~dB}$ SPL (L1) and $55 \mathrm{~dB}$ SPL (L2). Full details of the measuring equipment are described elsewhere [11, 12].

Cochlear microphonic potentials were recorded with two differential $\mathrm{Ni}-\mathrm{Cr}$ electrodes with a diameter of $50 \mu \mathrm{m}$. One electrode was placed in scala tympani through the bony wall of the basal turn of the cochlea. The other was placed in scala vestibuli next to the oval window. The signals were routed via an amplifier to a spectrum analyzer and two lock-in amplifiers (SRS, model SR830 DSP). The lock-in amplifiers displayed the amplitude of the $2 f_{1}-f_{2}$ and $f_{2}-f_{1}$ CMDP which were referenced to an electronic $2 f_{1}-f_{2}$ and $f_{2}-f_{1}$-DP derived from the original primaries.

A measurement contained a sequence of ten microinjections of artificial perilymph of $10 \mathrm{~s}$ each with pauses of $40 \mathrm{~s}$. The rate of microinjection was $50 \mathrm{nl} / \mathrm{s}$. The repetitive microinjection was controlled with a precision electronic timer (Stanford DG535). During an experiment, National Instruments LabVIEW ${ }^{\circledR}$ was used for recording of the following output signals: amplitude and phase of the $2 f_{1}-$ $f_{2}$ DPOAE, $2 f_{1}-f_{2}$ CMDP, $f_{2}-f_{1}$ CMDP, $f_{1}$ CM, and inner ear pressure. Calculations and fits to relevant portions of the obtained recordings were made off-line with an appropriate software package.

\section{Results}

Successful repeated sequences of injection with a rate of $50 \mathrm{nl} / \mathrm{s}(n=10)$ were performed in all guinea pigs $(n=8)$. The mean steady state inner ear pressure measured prior to injection of artificial perilymph was $205 \mathrm{~Pa}$.

To determine the rise and fall times of the curves from the measured signals, proper fits were obtained with a simple exponential function:

$a \exp (-t / \tau)+c$

In some cases (Figs. 1c, 2c, 4c) the curves needed an extra term in the formula for a proper fit:

$a \exp (-t / \tau) /[1+b\{1-\exp (-t / \tau)\}]+c$.

According to Wit et al. [13] time constants $\tau$ were calculated from the fits.

In Fig. 1a, the averaged recorded inner ear pressure during and after injection of $0.5 \mu \mathrm{l}$ of artificial perilymph is shown. At the start of injection the mean pressure increased 
Fig. 1 a Averaged changes $(n=8)$ in inner ear pressure $(\mathrm{Pa})$ during and after injection of $0.5 \mu \mathrm{l}$ of artificial perilymph into scala tympani. The gray area depicts the injection period. Standard error bars are shown at $t=5,15$, and $40 \mathrm{~s}$. b The shape of the mean inner ear pressure curve at the start of injection (time axis corresponds with Fig. 1a). The recording (filled circles) is fitted (broken line) with an exponential function, yielding a time constant $\tau$. c The same is done for inner ear pressure recovery directly after injection
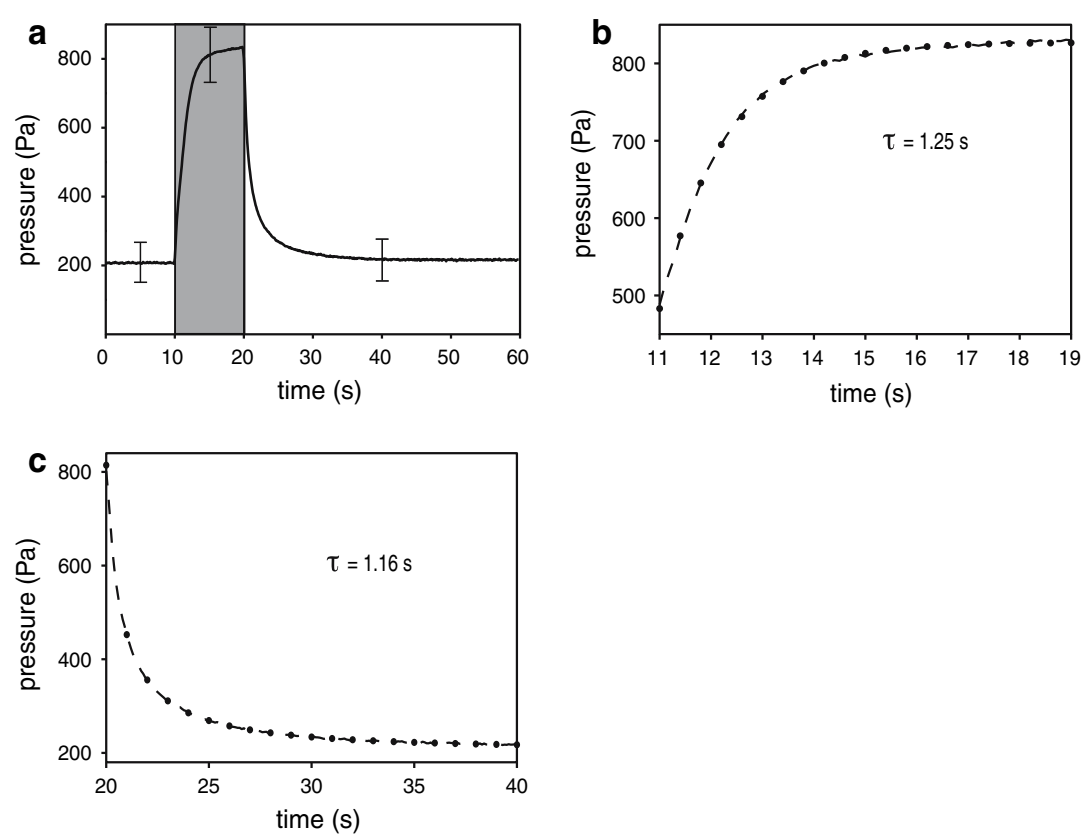

to an $\sim 600$ Pa higher level within seconds. At the end of injection, the pressure immediately decreased, returning to its initial value within seconds. This pattern was consistently observed in all individual experiments. The time constant for increase and subsequent decrease of inner ear pressure were 1.25 and $1.16 \mathrm{~s}$, respectively (Fig. 1b, c).

The averaged $f_{1}$ signal in the CM (Fig. 2a) showed a mirrored behavior in respect to the inner ear pressure. Directly after the start of injection, the $f_{1} \mathrm{CM}$ decreased by $30 \mu \mathrm{V}$. The corresponding time constant was $1.17 \mathrm{~s}$ (Fig. 2b), which is very close to the time constant for pressure increase. Conversely, at the end of injection the $f_{1}$ amplitude returned to its initial value with $\tau=3.49 \mathrm{~s}$ (Fig. 2c), which is slow with respect to the corresponding $\tau$ for pressure.

The averaged DPOAE $2 f_{1}-f_{2}$-amplitude showed a similar typical course as the inner ear pressure (Fig. 3a). Almost directly after the start of injection the $2 f_{1}-f_{2^{-}}$ amplitude increased to reach a maximum of $0.4 \mathrm{~dB}$ above its original value at the end of the injection period. When the injection was instantaneously terminated, the amplitude dropped and returned to slightly above its onset level. There was a small observable time lag with respect to the pressure curve. The time constant for increase and
Fig. 2 a Averaged changes $(n=8)$ in $f_{1} \mathrm{CM}$ amplitude $(\mu \mathrm{V})$ during and after injection of $0.5 \mu \mathrm{l}$ of artificial perilymph into scala tympani. The gray area depicts the injection period. Standard error bars are shown at $t=5,15$, and $40 \mathrm{~s}$. b The shape of the mean $f_{1} \mathrm{CM}$ amplitude curve at the start of injection (time axis corresponds with Fig. 2a). The recording (filled circles) is fitted (broken line) with an exponential function, yielding a time constant $\tau$. c The same is done for $f_{1} \mathrm{CM}$ amplitude recovery directly after injection
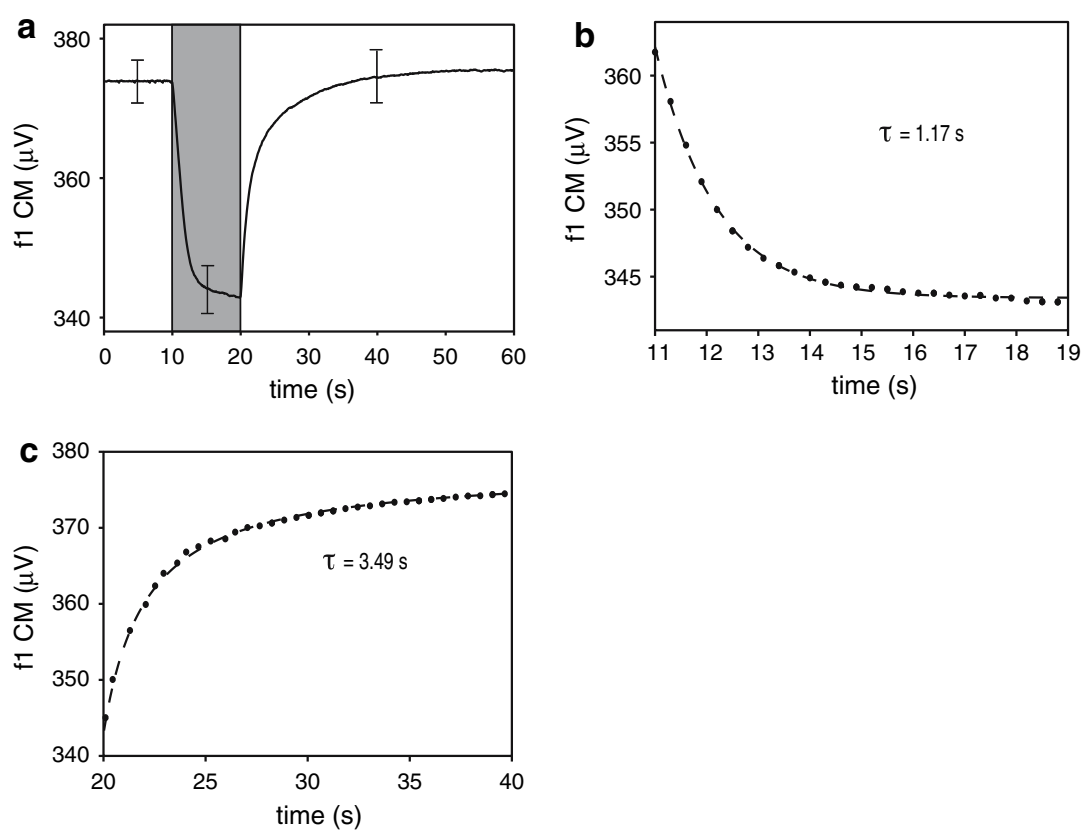
Fig. 3 a Averaged changes $(n=8)$ in $2 f_{1}-f_{2}$ DPOAE amplitude (dB SPL) during and after injection of $0.5 \mu \mathrm{l}$ of artificial perilymph into scala tympani. The gray area depicts the injection period. Standard error bars are shown at $t=5$, 15 , and $40 \mathrm{~s}$. b The shape of the mean $2 f_{1}-f_{2}$ DPOAE amplitude $(\mu \mathrm{Pa})$ curve at the start of injection (time axis corresponds with Fig. 3a). The recording (filled circles) is fitted (broken line) with an exponential function, yielding a time constant $\tau$. c The same is done for $2 f_{1}-f_{2}$ DPOAE amplitude recovery directly after injection
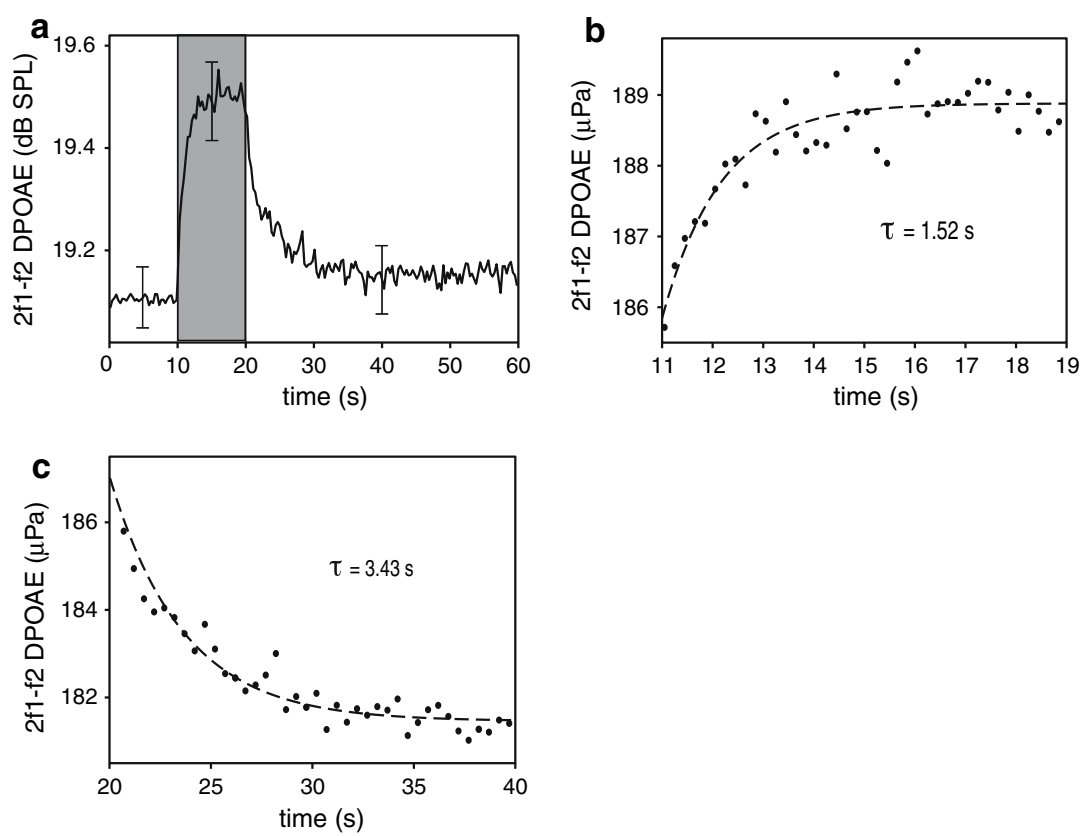

subsequent decrease of the $2 f_{1}-f_{2}$-amplitude were 1.52 and $3.43 \mathrm{~s}$, respectively (Fig. 3b,c).

In Fig. 4a, the typical temporary decrease for the averaged $2 f_{1}-f_{2}$ CMDP amplitude relative to the $f_{1} \mathrm{CM}$ is shown. The time constant for decrease in amplitude at the onset of injection was $1.12 \mathrm{~s}$ (Fig. 4b), which corresponds with $\tau$ for pressure. The recovery is somewhat slower (2.28 s, Fig. 4c), but still faster than the time constant for $2 f_{1}-f_{2}$ DPOAE recovery.

Finally, in Fig. 5, the averaged results of the $f_{2}-f_{1}$ CMDP amplitude relative to $f_{1} \mathrm{CM}$ depict an increase during injection of artificial perilymph. When the injection was terminated, the $f_{2}-f_{1}$ CMDP amplitude returned to the preinjection level. Unfortunately, due to a low signal-to-noise ratio for the $f_{2}-f_{1}$ CMDP, the obtained curve could not be fitted properly and no time constants could be derived.

\section{Discussion}

The most striking result of this study is the minimal change in amplitude of the DPs in both OAE and CM during an acute increase of inner ear pressure. Besides, amplitudes changed to normal when inner ear pressure returned to its
Fig. 4 a Averaged changes $(n=8)$ in $2 f_{1}-f_{2}$ CMDP relative to $f_{1} \mathrm{CM}$ amplitude during and after injection of $0.5 \mu \mathrm{l}$ of artificial perilymph into scala tympani. The gray area depicts the injection period. Standard error bars are shown at $t=5,15$, and $40 \mathrm{~s}$. b The shape of the mean $2 f_{1}-f_{2}$ CMDP (relative to $f_{1} \mathrm{CM}$ ) amplitude curve at the start of injection (time axis corresponds with Fig. 4a). The recording (filled circles) is fitted (broken line) with an exponential function, yielding a time constant $\tau$. c The same is done for $2 f_{1}-f_{2}$ CMDP (relative to $f_{1}$ $\mathrm{CM}$ ) amplitude recovery directly after injection
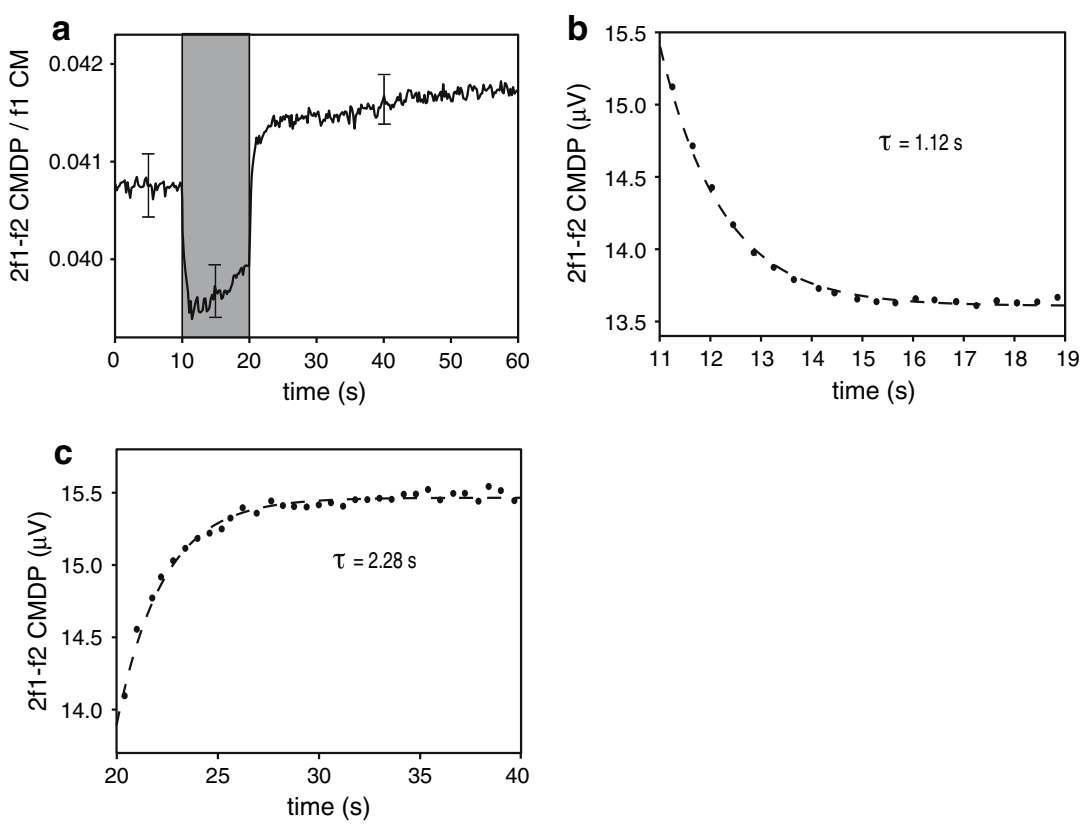


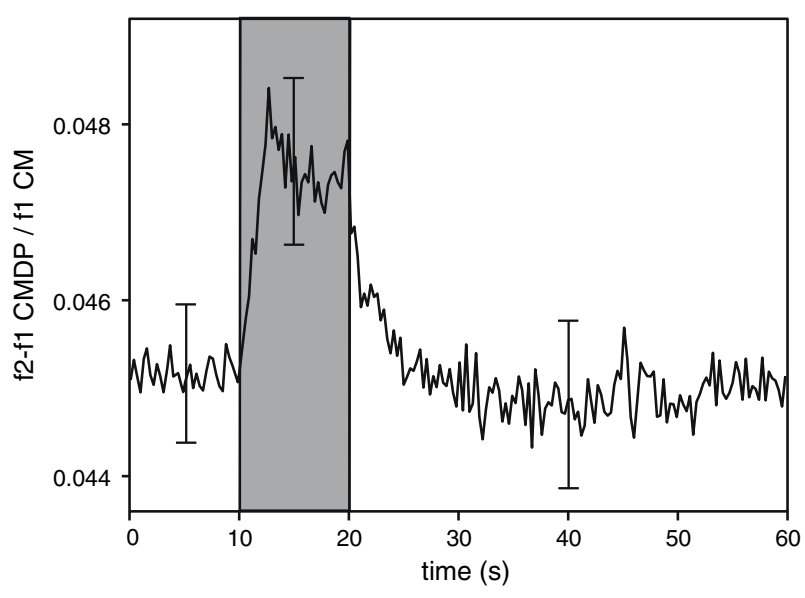

Fig. 5 Averaged changes $(n=8)$ in $f_{2}-f_{1}$ CMDP relative to $f_{1} \mathrm{CM}$ amplitude during and after injection of $0.5 \mu$ of artificial perilymph into scala tympani. The gray area depicts the injection period

initial level. These results indicate a dynamically stable inner ear which is able to cope with a pressure increase of $600 \mathrm{~Pa}$ with only minimal and reversible cochlear function loss. According to Böhmer [2] this level of pressure increase would still be in the physiological range of the guinea pig ear varying between -100 and $+700 \mathrm{~Pa}$.

The inner ear is an elastic structure completely encompassed by bone, in which pressure changes depend on the compliance of the membranous structures [14]. In normal ears, hydrostatic pressure in the perilymph equals pressure in the endolymph, and pressure changes applied to one compartment are immediately transmitted to the other through the elastic walls. The perilymph is in a dynamic equilibrium with the cerebrospinal fluid via the cochlear aqueduct. An excess of perilymph will escape through the cochlear aqueduct restoring inner ear fluid level.

When increasing inner ear pressure, a cochlear disturbance with functional loss is expected. The observed decrease in CM $f_{1}$ amplitude thus seems obvious. An increased cerebrospinal fluid pressure also suppresses the CM [16]. The increase in $2 f_{1}-f_{2}$ DPOAE is counterintuitive, but in accordance with a previous publication in which we demonstrated the same change in cochlear function as measured by $2 f_{1}-f_{2}$ DPOAE's [11]. According to Büki et al. [3] a change in stiffness of the oval window might slightly improve middle ear transfer and thus explain the small increase in DPOAE amplitude. In contrast to the $2 f_{1}-f_{2}$ DPOAE, the $2 f_{1}-f_{2}$ CMDP showed a decrease in amplitude during injection. Unfortunately, in literature very little can be found about the relation between DPOAE and CMDP. Still, it were Kemp and Brown [6] who found close parallels in the behavior of the $2 f_{1}-f_{2}$ DPOAE and CMDP during and after anoxia in the guinea pig. Group latencies and third-tone suppression were found to be very similar, linking both signals to have a common origin.
The DPOAE and CMDP are generated in response to the same acoustic stimuli. The acoustic primaries are transferred through the external and middle ear to eventually reach the sensory epithelia in the cochlea. The cochlea as a non-linear mechano-electrical transducer transforms sound pressure waves into electrical information. This forward transduction process is called the MET. The CM potential is a direct result of this process and is thought to be mainly produced by OHCs. On the other hand, there is also a reverse electromechanical transduction in which the $\mathrm{OHC}$ mechanically influences the vibration of the cochlear partition [7]. Sound is transmitted back to the external ear where the acoustic DPOAE can be measured; the backward transmission.

The rise and fall times of the DPs in response to changes in inner ear pressure were derived from the fitted curves (Figs. 2, 3, 4). As both CMDP and DPOAE were measured, this yielded information on forward and backward transmission. As anticipated, the time constants for the CMDP were smaller than the time constants for the DPOAE. Indeed, the latter would travel back and forth the middle ear which understandably costs more time. However, the difference has not the form of a simple longer delay. The explanation for this observation remains tentative. The averaged differences were 0.4 and $1.15 \mathrm{~s}$ for the $2 f_{1}-f_{2}$ DP during increase and decrease of inner ear pressure, respectively.

At pressure increase all measured signals almost directly followed the change in inner ear pressure. Time constants varied from 1.17 to $1.52 \mathrm{~s}$ with respect to $1.25 \mathrm{~s}$ for the inner ear pressure curve. However, with pressure decrease there was a delay of 1-2 s for all measured signals. Measured differences cannot be accounted for by delay in measuring equipment, for this was $<0.18 \mathrm{~s}$. Assumed that the overall stiffness of the stapes-oval window-system governs the behavior of the DPOAE $2 f_{1}-f_{2}$ amplitude, it is expected that it would follow stiffness changes directly and not $2 \mathrm{~s}$ later, as observed. It is conceivable that a delayed change in the endocochlear potential (EP) could be the cause of difference in $\tau$ values. However, in experiments by Salt and DeMott [9] the EP followed a temporary pressure change with a delay of $<0.1 \mathrm{~s}$. So, the mechanisms for the observed delays in inner ear pressure recovery remain to be elucidated.

Two-tone distortions are generated during non-linear mechanical amplification in the cochlea. Generation of the $2 f_{1}-f_{2}$ depends on asymmetric component of a non-linear transfer function whereas the $f_{2}-f_{1}$ depends on symmetric components. A change of the operating point is reflected in the level of the $f_{2}-f_{1}$ distortion. In the gerbil low-frequency tones of $5 \mathrm{~Hz}$ were used to bias the position of the cochlear partition by Frank and Kossl $[4,5]$.

By low-frequency modulation of the $2 f_{1}-f_{2}$ DPOAE, Bian et al. [1] described the forward MET by a sigmoid- 
shaped function, which related the displacement of the basilar membrane to the response of the hair cells.

By injecting artificial perilymph into scala tympani of the cochlea, the basilar membrane is temporarily displaced. Thus, by this displacement toward scala media, the operating state of the cochlear amplifier is also influenced. We measured a small decrease in the amplitude of the $2 f_{1}-f_{2}$ distortion in $\mathrm{CM}$ (with respect to the amplitude of $f_{1}$ in $\mathrm{CM}$ ) and a small increase in the $f_{2}-f_{1}$ in CM. Using the model by Bian et al. [1] this leads to the conclusion that the operating point for hair cell transduction is somewhat shifted from a symmetric position. This shift leads to an increase in even-order distortion and a decrease in oddorder distortion. Along these lines of reasoning we would also expect the $2 f_{1}-f_{2}$ DPOAE to decrease. This might be the case at the generation sites [15], but this effect is then more than compensated for by an improved middle ear transfer.

Anyhow, the observed changes are small. Changes of overall inner ear fluid pressure have minor effects on cochlear function. It may be that creating an artificial endolymphatic hydrops, by injecting artificial endolymph into scala media instead of scala tympani, has a larger effect. This is currently tested in further research, which will hopefully give insight into diseases with pathologically changed inner ear fluid volumes, as in Ménière's disease.

Acknowledgments This study was supported by the Heinsius Houbolt Foundation and is part of the research program of our department: Communication through Hearing and Speech. The program is incorporated in the Sensory Systems Group of the Groningen Graduate School for Behavioural and Cognitive Neurosciences (BCN). We thank Hans Segenhout and Wilma Hiemstra for their invaluable contribution in performing the experimental work in this study.

\section{References}

1. Bian L, Chertoff ME, Miller E (2002) Deriving a cochlear transducer function from low-frequency modulation of distortion product otoacoustic emissions. J Acoust Soc Am 112:198-210
2. Böhmer A (1994) Hydrostatic pressure in the inner ear fluid compartments and its effects on inner ear function. Acta Otolaryngol Suppl 507:3-24

3. Büki B, Avan P, Lemaire JJ, Dordain M, Chazal J, Ribari O (1996) Otoacoustic emissions: a new tool for monitoring intracranial pressure changes through stapes displacements. Hear Res 94:125-139

4. Frank G, Kossl M (1996) The acoustic two-tone distortions $2 f_{1}-$ $f_{2}$ and $f_{2}-f_{1}$ and their possible relation to changes in the operating point of the cochlear amplifier. Hear Res 98:104-115

5. Frank G, Kossl M (1997) Acoustical and electrical biasing of the cochlear partition. Effects on the acoustic two tone distortions $f_{2}-f_{1}$ and $2 f_{1}-f_{2}$. Hear Res 113:57-68

6. Kemp DT, Brown AM (1984) Ear canal acoustic and round window electrical correlates of $2 f_{1}-f_{2}$ distortion generated in the cochlea. Hear Res 13:39-46

7. Kim DO (1986) Active and nonlinear cochlear biomechanics and the role of outer-hair-cell subsystem in the mammalian auditory system. Hear Res 22:105-114

8. de Kleine E, Wit HP, van Dijk P, Avan P (2000) The behavior of spontaneous otoacoustic emissions during and after postural changes. J Acoust Soc Am 107:3308-3316

9. Salt AN, DeMott JE (1998) Longitudinal endolymph movements induced by perilymphatic injections. Hear Res 123:137-147

10. Salt AN, DeMott JE (1999) Longitudinal endolymph movements and endocochlear potential changes induced by stimulation at infrasonic frequencies. J Acoust Soc Am 106:847-856

11. Valk WL, Wit HP, Albers FW (2004) Effect of acute inner ear pressure changes on low-level distortion product otoacoustic emissions in the guinea pig. Acta Otolaryngol 124:929-936

12. Valk WL, Wit HP, Albers FW (2004) Evaluation of cochlear function in an acute endolymphatic hydrops model in the guinea pig by measuring low-level DPOAEs. Hear Res 192:47-56

13. Wit HP, Thalen EO, Albers FW (1999) Dynamics of inner ear pressure release, measured with a double-barreled micropipette in the guinea pig. Hear Res 132:131-139

14. Wit HP, Warmerdam TJ, Albers FW (2000) Measurement of the mechanical compliance of the endolymphatic compartments in the guinea pig. Hear Res 145:82-90

15. Withnell RH, Shaffer LA, Talmadge CL (2003) Generation of DPOAEs in the guinea pig. Hear Res 178:106-117

16. Yoshida M, Uemura T (1991) Transmission of cerebrospinal fluid pressure changes to the inner ear and its effect on cochlear microphonics. Eur Arch Otorhinolaryngol 248:139-143

17. Zhang M, Abbas PJ (1997) Effects of middle ear pressure on otoacoustic emission measures. J Acoust Soc Am 102:1032-1037 